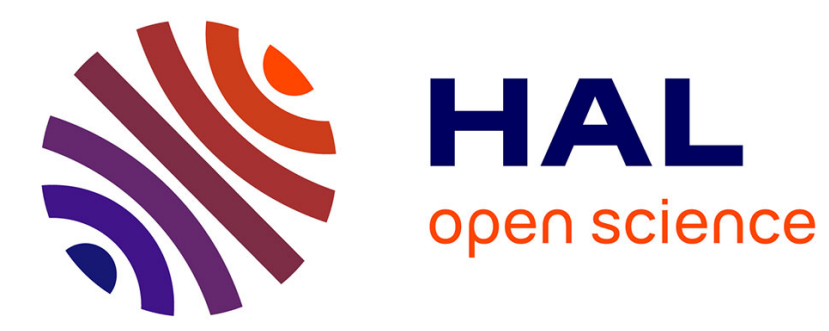

\title{
Transit spectroscopy of temperate Jupiters with ARIEL: a feasibility study
}

\author{
Thérèse Encrenaz, G. Tinetti, A. Coustenis
}

\section{To cite this version:}

Thérèse Encrenaz, G. Tinetti, A. Coustenis. Transit spectroscopy of temperate Jupiters with ARIEL: a feasibility study. Experimental Astronomy, 2018, 46 (1), pp.31-44. 10.1007/s10686-017-9561-2 . hal-01992793

\section{HAL Id: hal-01992793 https://hal.sorbonne-universite.fr/hal-01992793}

Submitted on 24 Jan 2019

HAL is a multi-disciplinary open access archive for the deposit and dissemination of scientific research documents, whether they are published or not. The documents may come from teaching and research institutions in France or abroad, or from public or private research centers.
L'archive ouverte pluridisciplinaire HAL, est destinée au dépôt et à la diffusion de documents scientifiques de niveau recherche, publiés ou non, émanant des établissements d'enseignement et de recherche français ou étrangers, des laboratoires publics ou privés. 


\title{
Transit spectroscopy of temperate Jupiters with ARIEL: A feasibility study
}

\author{
T. Encrenaz ${ }^{1}, \mathrm{G}$. Tinetti ${ }^{2}$ and A. Coustenis ${ }^{1}$ \\ ${ }^{1}$ LESIA, Paris Observatory, CNRS, PSL Universities, UPMC, UDD \\ 2 Department of Physics and Astronomy, University College London
}

Submitted to Experimental Astronomy

ARIEL Special Issue

Revised version

September 2017 


\section{Corresponding author:}

Thérèse Encrenaz

LESIA, Observatoire de Paris, CNRS, PSL Universities, UPMC, UDD

Observatoire de Paris

5 place Janssen

92195 Meudon, France

Tel: 33145077691

Fax: 33145072806

e-mail: therese.encrenaz@obspm.fr 


\begin{abstract}
Several temperate Jupiters have been discovered to date, but most of them remain to be detected. In this note, we analyse the expected infrared transmission spectrum of a temperate Jupiter, with an equilibrium temperature ranging between 350 and $500 \mathrm{~K}$. We estimate its expected amplitude signal through a primary transit, and we analyse the best conditions for the host star to be filled in order to optimize the $\mathrm{S} / \mathrm{N}$ ratio of its transmission spectrum. Calculations show that temperate Jupiters around M stars could have an amplitude signal higher than $10^{-4}$ in primary transits, with revolution periods of a few tens of days and transit durations of a few hours. In order to enlarge the sampling of exoplanets to be observed with ARIEL (presently focussed on objects warmer than $500 \mathrm{~K}$ ), such objects could be considered as additional possible targets for the mission.
\end{abstract}




\section{Introduction}

Temperate Jupiters, with equilibrium temperatures ranging between 350 and $500 \mathrm{~K}$, are not expected to exist according to the standard nucleation model, which requires that giant planets are formed at large distances from their host star, at temperatures low enough for water and other hydrogenated molecules to be in the form of ices (see e.g. Pollack et al. 1996). However, several temperate exoplanets with radii larger than half the Jupiter one and with periods ranging between 100 and 300 days have been detected around $F, G$ and $K$ stars (some examples are listed in Table 1, for semi-major axes ranging between 0.5 and $1.0 \mathrm{AU}$ an eccentricities smaller than 0.1 ). It can be seen that their equilibrium temperatures range between 250 and $500 \mathrm{~K}$. Their presence at relatively close distances to their host star might be the result of migration processes, or other possible formation mechanisms, still to be investigated. Although most of the objects detected so far are not suitable or probably too faint for transit spectroscopy, their detection demonstrates that this category of objects actually exists. If such objects are also transiting around low-mass stars, they would be suitable targets for transit spectroscopy, in particular with the ARIEL space mission. Future surveys with space missions like GAIA (Global Astrometric Interferometer for Astrophysics), but also CHEOPS (CHaracterizing ExOPlanets Satellite), TESS (Transiting Exoplanet Survey Satellite) or PLATO (PLAnetary Transits and Oscillations of stars), are likely to provide new targets for this category of exoplanets.

\section{Table 1}

Examples of temperate giant exoplanets detected around $\mathrm{F}, \mathrm{G}$ and $\mathrm{K}$ stars, with a mass larger than 0.5 Jovian mass and an eccentricity smaller than 0.1 (from www.exoplanets.eu). The equilibrium temperature is calculated assuming an albedo $\mathrm{a}=$ 0.03 and a fast-rotating planet (Equation 4, see text below, Section 2).

\begin{tabular}{|l|l|l|l|l|l|l|l|}
\hline Name & $\mathrm{M}_{\mathrm{P}}\left(\mathrm{M}_{\mathrm{J}}\right)$ & $\mathrm{P}(\mathrm{d})$ & $\mathrm{D}(\mathrm{AU})$ & $\mathrm{e}$ & $\begin{array}{l}\text { Spectral } \\
\text { type }\end{array}$ & $\begin{array}{l}\mathrm{M}^{*} \\
(\mathrm{Ms})\end{array}$ & $\begin{array}{l}\mathrm{T}_{\mathrm{P}} \\
(\mathrm{K})\end{array}$ \\
\hline HD 134113 b & 47 & 202 & 0.64 & 0.089 & $\mathrm{~F} 9 \mathrm{~V}$ & 0.81 & 295 \\
\hline HD 233604 b & 6.6 & 192 & 0.747 & 0.05 & K5 & 1.5 & 434 \\
\hline HD 28185 b & 5.7 & 383 & 1.03 & 0.07 & G5 & 1.24 & 320 \\
\hline HD 32518 b & 3.04 & 157 & 0.59 & 0.01 & K1 III & 1.13 & 395 \\
\hline HD 159243 c & 1.9 & 248 & 0.8 & 0.075 & G0 V & 1.125 & 338 \\
\hline HD 9446 c & 1.82 & 193 & 0.654 & 0.06 & G5 V & 1.0 & 342 \\
\hline HD 141399 c & 1.33 & 202 & 0.69 & 0.048 & K0 V & 1.07 & 390 \\
\hline HD 231701 b & 1.08 & 142 & 0.53 & 0.096 & F8 V & 1.14 & 419 \\
\hline Kepler-11 g & 0.95 & 118 & 0.46 & 0.0 & G & 0.95 & 392 \\
\hline HD 92788 c & 0.9 & 162 & 0.6 & 0.04 & G5 & 1.13 & 392 \\
\hline HD 37124 b & 0.675 & 154 & 0.53 & 0.054 & G4 V & 0.83 & 331 \\
\hline HD 45364 c & 0.66 & 343 & 0.897 & 0.097 & K0 V & 0.82 & 252 \\
\hline Mu Ara d & 0.52 & 310 & 0.92 & 0.07 & G3 IV-V & 1.08 & 306 \\
\hline
\end{tabular}

In this paper, we investigate what could be the infrared spectrum of a temperate Jupiter and we study its detectability with the ARIEL space mission using transit spectroscopy, 
both through primary transit and secondary transit. We then analyse the most favorable type of star in order to optimize the primary transit signal.

The objective of ARIEL is the characterization of a large sample of exoplanets'atmospheres (500 - 1000 targets) using transit spectroscopy in the midinfrared range $(2-8 \mu \mathrm{m})$, with a resolving power between 100 and 200 . It consists in an off-axis $90-\mathrm{cm}$ telescope, passively cooled at 60-70 K, with a spectrometer cooled at $50 \mathrm{~K}$ and MCT detectors also cooled at $40 \mathrm{~K}$. The stellar light is simultaneously recorded by a photometer operating at $1-2 \mu \mathrm{m}$. ARIEL is primarily designed for observing various kinds of exoplanets with equilibrium temperatures higher than $500 \mathrm{~K}$. In order to enlarge the diversity of targets observable with ARIEL, we analyze in this paper the feasibility of observing cooler giant exoplanets, with equilibrium temperatures ranging between 350 and $500 \mathrm{~K}$.

\section{The equilibrium temperature of an exoplanet}

The equilibrium temperature $\mathrm{T}_{\mathrm{e}}$ of a slow-rotating exoplanet is given by this equation:

$$
\begin{aligned}
& \left.\mathrm{T}_{\mathrm{e}}=(1-\mathrm{a})^{0.25} \times 331.0 \times\left(\mathrm{T}^{*} / 5770\right) \times \mathrm{R}^{* 0.5}\right) / \mathrm{D}^{0.5} \\
& \text { or } \\
& \left.\mathrm{T}_{\mathrm{e}}=(1-\mathrm{a})^{0.25} \times 331.0 \times\left(\mathrm{M}^{*}\right)^{3 / 4}\right) / \mathrm{D}^{0.5}
\end{aligned}
$$

where $\mathrm{a}$ is the albedo, $\mathrm{T}^{*}$ the effective temperature of the star (in $\left.\mathrm{K}\right), \mathrm{R}^{*}$ its radius and $\mathrm{M}^{*}$ its mass (in solar units), and D the distance of the exoplanet to the star (in AU). For a fast-rotating exoplanet, these equations become:

$$
\begin{aligned}
& \left.\mathrm{T}_{\mathrm{e}}=(1-\mathrm{a})^{0.25} \times 279.0 \times\left(\mathrm{T}^{*} / 5770\right) \times \mathrm{R}^{* 0.5}\right) / \mathrm{D}^{0.5} \\
& \text { or } \\
& \left.\mathrm{T}_{\mathrm{e}}=(1-\mathrm{a})^{0.25} \times 279.0 \times\left(\mathrm{M}^{*}\right)^{3 / 4}\right) / \mathrm{D}^{0.5}
\end{aligned}
$$

In the case of a solar type star, taking as extreme conditions albedo values of 0.3 and 0.03 (as most commonly observed in the solar system), we find distances to the host star ranging between $0.26 \mathrm{AU}$ (for a fast-rotating planet, $\mathrm{T}_{\mathrm{e}}=500 \mathrm{~K}$ and $\mathrm{a}=0.3$ ) and $0.9 \mathrm{AU}$ (for a slow-rotating planet, $\mathrm{T}_{\mathrm{e}}=350 \mathrm{~K}$ and $\mathrm{a}=0.03$ ). For a solar-type star, the corresponding revolution periods range between 48 days and 312 days. The periods decrease if low-mass stars are considered. In order to perform transit spectroscopy of temperate Jupiters, several transits have to be performed over the lifetime of the mission, so, in what follows, we will focus on stars less massive than the Sun which allow a larger number of transits.

In order to estimate the equilibrium temperature of an exoplanet, we need to make an assumption on its rotation period. As a first approximation, this can be estimated from a comparison of its distance to the star versus the tidal lock radius, i.e. the distance to the star within which synchronous rotation takes place as a result of tidal forces. According 
to Murray and Dermott (1999) and Léger et al. (2009), the characteristic time for a planet to reach synchronous rotation is given by:

$\tau_{\text {synch }}=\left|\left(n-\Omega_{P}\right)\right| \times M_{P} \times D^{6} \times I \times Q / k_{2 P} /\left[3 / 2 \times G \times R_{P}^{3} \times M^{* 2}\right]$

where $\mathrm{n}$ is the mean motion of the revolution rate of the planet, $\Omega$ is its rotation rate, I is its moment of inertia, $\mathrm{Q}$ its dissipation constant, $\mathrm{k}_{2 \mathrm{P}}$ its Love number of second order. $\mathrm{M}_{\mathrm{P}}$ is the planetary mass and $R_{P}$ is its radius, $D$ is the distance of the planet to the star, $M^{*}$ is the stellar mass and $\mathrm{G}$ is the gravitational constant. It can be seen that for a given planet, $\tau_{\text {synch }}$ is proportional to $\mathrm{D}^{6} / \mathrm{M}^{* 2}$. Thus, the tidal lock radius $\mathrm{D}_{\text {synch, corresponding to a }}$ given value of $\tau_{\text {synch, }}$ is proportional to $\left[\mathrm{M}^{*}\right]^{1 / 3}$. This is shown in the study by Kasting et al. (1993; Fig. 16) who indicate the tidal lock radius for Earth-like exoplanets; they find a tidal-lock radius $\mathrm{D}_{\text {synch }}$ of $0.4 \mathrm{AU}$ for a solar-mass star and $0.2 \mathrm{AU}$ for a star of 0.1 solar mass. The corresponding revolution period of the planet is about 90 days, independent of the stellar mass.

For the present study, we need to estimate the tidal lock radius for giant exoplanets. In the lack of information about the values of the tidal parameters of these planets, we can compare the physical and tidal parameters of giant solar system planets as compared with terrestrial ones. Planetary moments of inertia range between 0.2 and 0.35 . Rotation periods of the Earth and Jupiter are within a factor of 2 . The $Q / \mathrm{k}_{2 \mathrm{P}}$ factor is about 1000 for the Earth while this quantity is in the range $10^{3}-10^{5}$ for Jupiter and Saturn (Lainey, 2006). This leads to a $\tau_{\text {synch }}$ value between 1 and 100 times the terrestrial value for giant exoplanets. Accordingly, $\mathrm{D}_{\text {synch }}$ is expected to range between the terrestrial value (as indicated by Kasting et al. 1993) and half this value, and the corresponding limit for the revolution period is between 90 days and 33 days. As a consequence, the values of $\mathrm{T}_{\mathrm{e}}$ shown in Table 1 are calculated using Equation 4, corresponding to fast-rotating exoplanets.

\section{The atmosphere of a temperate Jupiter}

Let us assume a Jupiter-type planet with an equilibrium temperature ranging between $350 \mathrm{~K}$ and $500 \mathrm{~K}$ under thermochemical equilibrium. The atmosphere is expected to be characterized by a troposphere where the temperature decreases as the altitude increases, following the adiabatic gradient. The atmospheric composition is expected to be dominated by hydrogen and helium. As for solar system giant planets, we assume for the tropopause a pressure level of 0.1 bar, constrained by the hydrogen and helium opacities. Figure 1 shows a $\mathrm{P}(\mathrm{T})$ diagram of a temperate Jupiter. If we assume thermochemical equilibrium, it can be seen that, in the exoplanet's environment, nitrogen and carbon are expected to be in the form of $\mathrm{NH}_{3}$ and $\mathrm{CH}_{4}$ respectively. In the same way, oxygen is expected in the reduced form of water $\mathrm{H}_{2} \mathrm{O}$. 


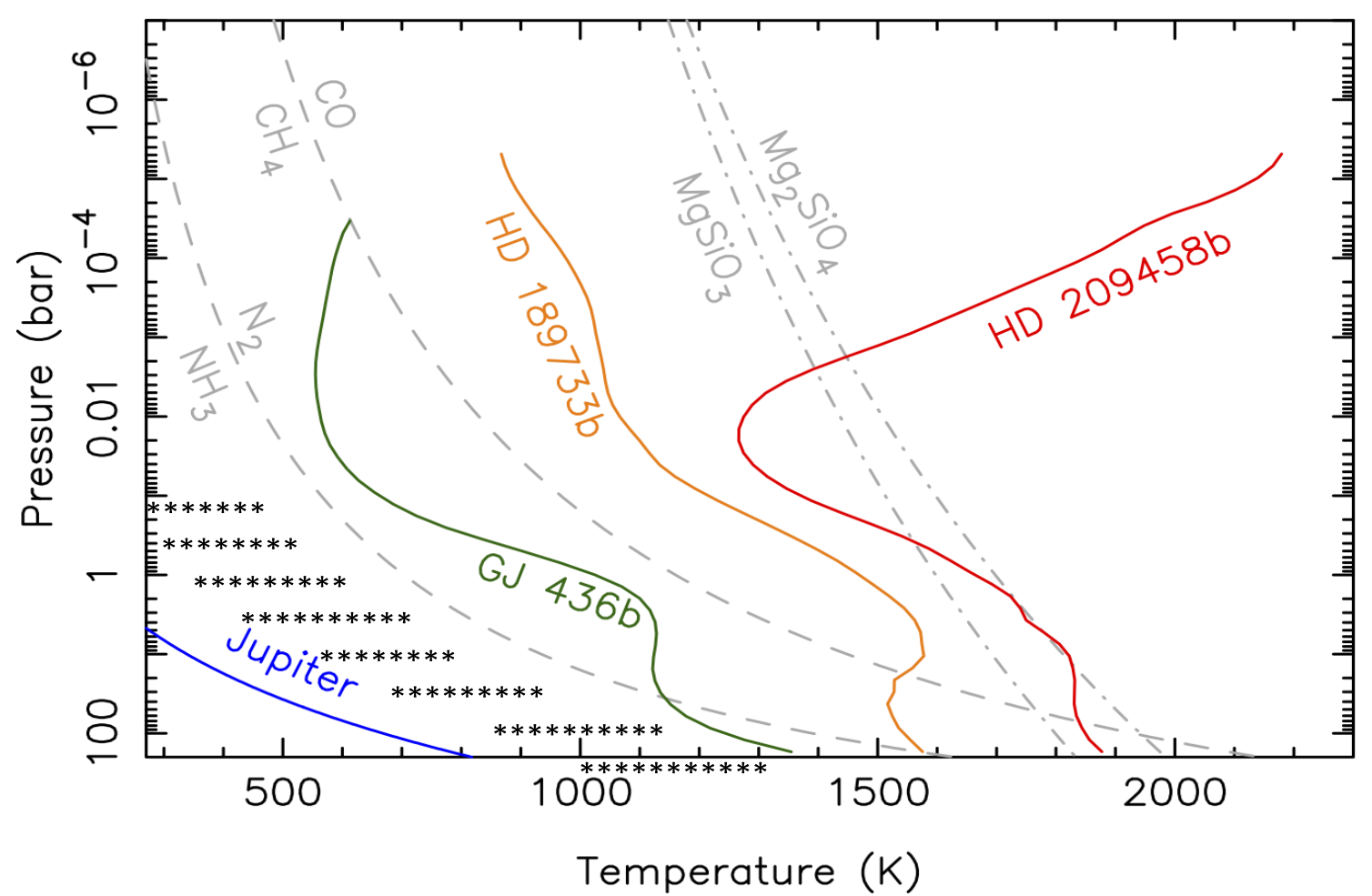

Figure 1. The $T(P)$ diagram of a temperate Jupiter with an equilibrium temperature ranging between $350 \mathrm{~K}$ and $500 \mathrm{~K}$. The range corresponding to temperate Jupiters is shown with the black dots. It can be seen that carbon and nitrogen are expected to be in the form of $\mathrm{NH}_{3}$ and $\mathrm{CH}_{4}$ respectively. The figure is adapted from the EChO proposal (Tinetti et al. 2011).

In the troposphere, according to thermochemical equilibrium, $\mathrm{CH}_{4}, \mathrm{NH}_{3}$ and $\mathrm{H}_{2} \mathrm{O}$ are expected to be the main minor species. We assume for them, in the troposphere, the cosmic abundances. We know, however, that in the case of the giant planets of the solar system, these molecules are enriched by a factor about 4 as a result of the nucleation formation Owen and Encrenaz 2006). This factor of 4 depends upon the relative mass of the initial icy core with respect to the total mass of the planet (3\% in the case of Jupiter); in the case of Saturn, for which the initial icy core is about $15 \%$ of the total mass, the expected enrichment is a factor of about 9 . When the icy core becomes a major part of the total mass of the giant planet, as for Uranus and Neptune, the enrichment factor is above 30-40. We note that, in any case, in the nucleation model, the enrichments are expected to be the same for $\mathrm{O}, \mathrm{C}$, and N; so we can assume that the relative abundances of $\mathrm{CH}_{4}, \mathrm{NH}_{3}$ and $\mathrm{H}_{2} \mathrm{O}$ in the troposphere of the exoplanet are consistent with the cosmic abundances (Grevesse et al. 2005). In our calculations, we assume for $\mathrm{CH}_{4}$ and $\mathrm{NH}_{3}$ the same abundances as measured in Jupiter below the condensation levels: $\mathrm{CH}_{4} / \mathrm{H}_{2}=2 \mathrm{x}$ $10^{-3}$ (Encrenaz et al. 1999), $\mathrm{NH}_{3} / \mathrm{H}_{2}=2 \times 10^{-4}$ (Encrenaz et al. 1999, Bolton et al. 2017). For $\mathrm{H}_{2} \mathrm{O}$ we assume $\mathrm{H}_{2} \mathrm{O} / \mathrm{H}_{2}=4 \times 10^{-3}$ below the water cloud level. We thus adopt $\mathrm{H}_{2} \mathrm{O}: \mathrm{CH}_{4}: \mathrm{NH}_{3}=2: 1: 0.1$. It must be noted that these numbers are no more than examples, chosen to build a typical spectrum of a temperate Jupiter.

With respect to the true atmosphere of Jupiter, the main difference of a temperate Jupiter is that there is no cold trap at the tropopause: at a temperature above $350 \mathrm{~K}$, all molecules, including $\mathrm{H}_{2} \mathrm{O}$ and $\mathrm{NH}_{3}$, keep a constant mixing ratio with the altitude, up to a 
level where they become photo-dissociated. As a result, the infrared spectrum of a temperate Jupiter is expected to be fully dominated by $\mathrm{CH}_{4}, \mathrm{NH}_{3}$ and $\mathrm{H}_{2} \mathrm{O}$.

Our transmission spectra are generated using a line-by-line calculation of the absorption coefficient, assuming a single pressure layer (1 bar) and a single temperature $(300 \mathrm{~K})$. We use for $\mathrm{NH}_{3}$ a column density of 3 m-amagat, as measured in Jupiter in the $1.56-\mu \mathrm{m}$ spectral window which probes down to a pressure of a few bars (Owen et al. 1977); we then derive column densities of $30 \mathrm{~m}$-amagat for $\mathrm{CH}_{4}$ and $60 \mathrm{~m}$-amagat for $\mathrm{H}_{2} \mathrm{O}$, respectively. Our transmission spectrum is convolved with a Gaussian function with a resolving power of 100 at $2 \mu \mathrm{m}, 200$ at $4 \mu \mathrm{m}$ and 400 at $8 \mu \mathrm{m}$. The resolution is comparable to the ARIEL one in the $2-4-\mu \mathrm{m}$ range and higher at $4-8 \mu \mathrm{m}$ range, which allows a better description of the band shapes.

Figure 2 shows the synthetic transmission spectrum of a temperate Jupiter. For comparison, Figure 3 shows the synthetic spectrum of a cold Jupiter, where most of the water is trapped in the form of ice in the lower troposphere (in the gaseous phase, $\left.\mathrm{H}_{2} \mathrm{O}: \mathrm{CH}_{4}: \mathrm{NH}_{3}=0.07: 1: 0.1\right)$. This corresponds to the spectrum of the actual Jupiter if it were seen in transmission in front of the Sun.

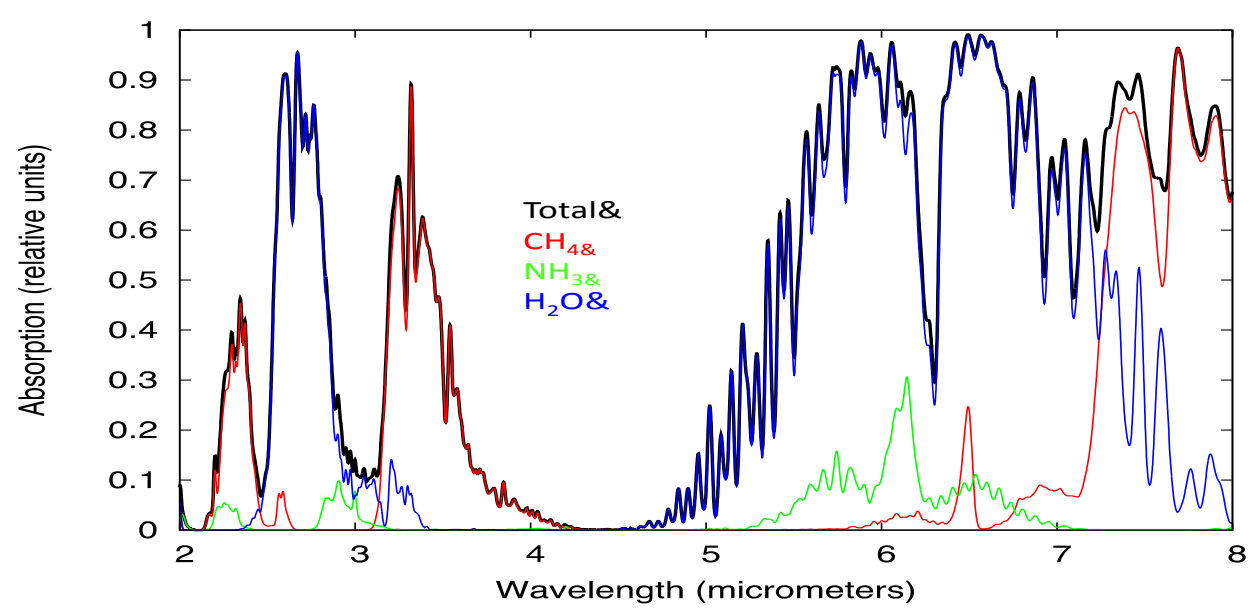

Figure 2. A typical transmission spectrum for a temperate Jupiter ( $\mathrm{T}=350 \mathrm{~K})$. Jupiter-like relative abundances are assumed for the molecules in the troposphere below the clouds, and no condensation is assumed for $\mathrm{H}_{2} \mathrm{O}: \mathrm{H}_{2} \mathrm{O}: \mathrm{CH}_{4}: \mathrm{NH}_{3}=2.0: 1.0: 0.1$. The full scale is equal to the amplitude of the transmission signal calculated in Table 2. 


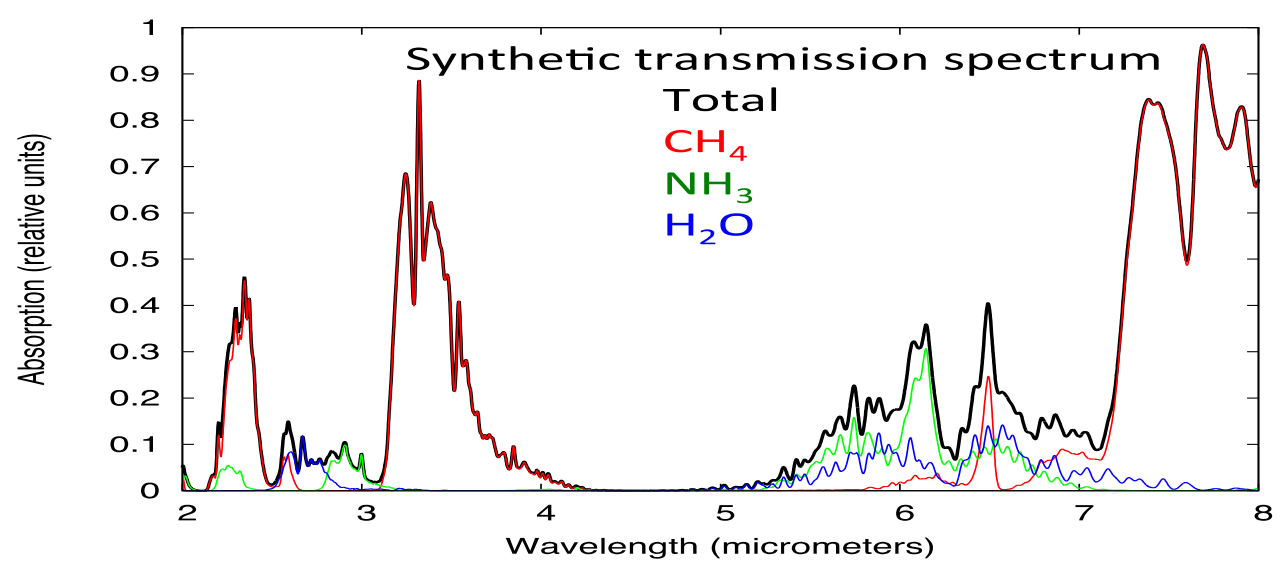

Figure 3. A typical transmission spectrum for a cold Jupiter $(T=150 \mathrm{~K})$. Jupiter-like relative abundances are assumed for the molecules in the troposphere, and condensation is assumed for $\mathrm{H}_{2} \mathrm{O}: \mathrm{H}_{2} \mathrm{O}: \mathrm{CH}_{4}: \mathrm{NH}_{3}=0.07: 1.0: 0.1$. The full scale is equal to the amplitude of the transmission signal calculated in Table 2.

Above the tropopause, other minor molecules are expected as products of photodissociation: $\mathrm{C}_{2} \mathrm{H}_{2}$ and $\mathrm{C}_{2} \mathrm{H}_{6}$ from the methane photo-dissociation, $\mathrm{CO}$ and $\mathrm{CO}_{2}$ from the water photo-dissociation. By analogy with the case of Jupiter, we estimate the possible contribution due to photochemistry in the transmission spectrum by assuming the following abundances: (1) at $\mathrm{P}=100 \mathrm{mb}$ (tropopause), $\mathrm{H}_{2} \mathrm{O}: \mathrm{CH}_{4}: \mathrm{NH}_{3}=2: 1: 0.1$ (as in the troposphere); (2) in the stratosphere, at $\mathrm{P}=1 \mathrm{mb}, \mathrm{CH}_{4}: \mathrm{C}_{2} \mathrm{H}_{2}: \mathrm{C}_{2} \mathrm{H}_{6}=1: 10^{-4}: 210^{-3}$, and $\mathrm{H}_{2} \mathrm{O}: \mathrm{CO}: \mathrm{CO}_{2}=1: 1: 0.2$. Calculations show that $\mathrm{CO}$ and $\mathrm{CO}_{2}$ contribute as minor absorbers in the infrared spectrum, while the contribution of hydrocarbons is negligible (Figure 4).

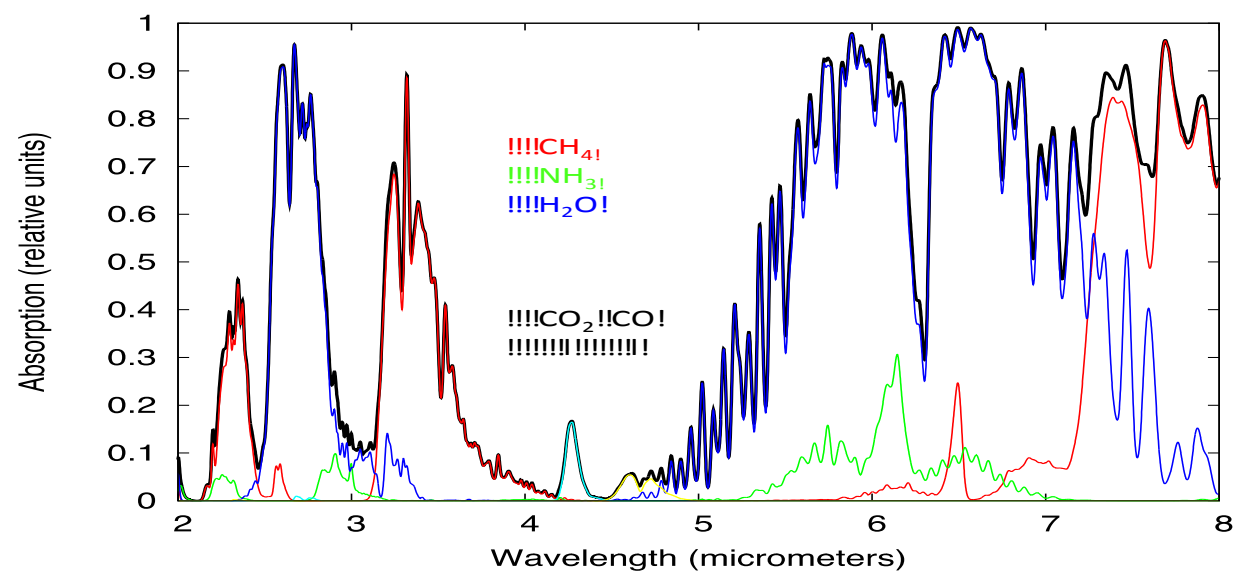

Figure 4. A typical transmission spectrum for a temperate giant exoplanet $(\mathrm{T}=350 \mathrm{~K})$, including the effect of photochemistry. Jupiter-like relative abundances are assumed for the molecules in the troposphere and no condensation is assumed $\left(\mathrm{P}=100 \mathrm{mb}, \mathrm{H}_{2} \mathrm{O}: \mathrm{CH}_{4}: \mathrm{NH}_{3}=\right.$ 2.0:1.0:0.1). In the stratosphere $(P=1 \mathrm{mb})$, the relative abundances are $\mathrm{H}_{2} \mathrm{O}: \mathrm{CO}: \mathrm{CO}_{2}=$ 1:1:0.2. The contribution of hydrocarbons is negligible. The full scale is equal to the amplitude of the transmission signal calculated in Table 2. 
What could be the condensates present in a temperate Jupiter? We know that hazes may be important contributors that flatten the infrared transmission spectrum of hot or warm exoplanets, as illustrated by the flat spectrum of GJ1214 b. For temperate objects colder than $500 \mathrm{~K}$, all ices are in gaseous form. We can thus expect temperate Jupiters to be relatively free of condensates, as discussed by Sudarsky et al. (2000). Morley et al. (2012), however, have shown that some condensates (including $\mathrm{Na} 2 \mathrm{~S}, \mathrm{ZnS}$ and $\mathrm{KCl}$ ) might be present in the atmospheres of T-dwarfs, so the possible presence of clouds in temperate exoplanets is still an open question. In the lack of more information, we adopt in our study a low value of the albedo (0.03), as has been actually observed on hot Jupiters (Rowe et al. 2008).

Our analysis is consistent with the study of Sudarsky et al. (2000) who have characterized the various types of giant exoplanets as a function of their equilibrium temperature. Giant exoplanets in the 350 - $500 \mathrm{~K}$ range, as considered in the present study, are intermediate between Class II and Class III and show no $\mathrm{NH}_{3}$ nor $\mathrm{H}_{2} \mathrm{O}$ clouds; they are supposed to have a very low albedo. The situation would be different for objects with an equilibrium temperature of $250 \mathrm{~K}$ (Class II), as the presence of a water cloud would lead to a high albedo. However, Class II objects are not considered in the present study because their expected transmission signal would be below the sensitivity limit of ARIEL (see Section 7).

\section{Primary transit spectroscopy of a temperate Jupiter}

Let us assume a Jupiter-size exoplanet transiting in front of its host star. During a primary transit, its atmosphere is observed in transmission at terminator in front of the stellar light. The area of planetary atmosphere observed in transmission is an annulus around the planet with a radial height of about $5 \times \mathrm{H}$, where $\mathrm{H}$ is the scale height. $\mathrm{H}$ is equal to $\mathrm{RT} / \mu \mathrm{g}$, where $\mathrm{R}$ is the perfect gaz constant, $\mathrm{T}$ the temperature, $\mu$ the mean molecular weight of the atmosphere and g the planet's gravity. The amplitude of the absorption within the exoplanet's atmosphere can be approximated as follows (Tinetti et al. 2013)

$\mathrm{A}=5 \times\left[2 \mathrm{RpH}_{\mathrm{p}} / \mathrm{R}^{* 2}\right]$

where $\mathrm{R}_{\mathrm{p}}$ and $\mathrm{R}^{*}$ are the radii of the planet and the star respectively.

For a hydrogen - helium rich planet with $\mu=2.4$, the scale height (in $\mathrm{km}$ ) can be expressed as

$\mathrm{H}=3.46 \times \mathrm{T}_{\mathrm{P}} / \mathrm{g}$

where $T_{P}$ is the equilibrium temperature of the exoplanet in Kelvins and $g$ is the surface gravity in $\mathrm{m} / \mathrm{s}^{2}$ (at a pressure level of 1 bar). The surface gravity g can be expressed as

$\mathrm{g}=25 \times \mathrm{MP} / \mathrm{RP}^{2}$ 
where Mp and Rpare the mass and the radius of the planet, expressed in Jovian masses and radii, respectively. As a result, the amplitude of the absorption can be written (with $\mathrm{R}^{*}$ expressed in solar radii):

$A=1.410^{-6} \times \operatorname{RP} \times H / R^{* 2}$

For a hydrogen-rich exoplanet, we have

$\mathrm{A}=1.9410^{-7} \times \mathrm{T} \times \mathrm{R}_{\mathrm{P}}^{3} / \mathrm{M}_{\mathrm{P}} / \mathrm{R}^{* 2}=1.9410^{-7} \times \mathrm{T} / \rho \mathrm{R}^{* 2}$

where $\rho$ is the planet's density, expressed in Jovian units. For hot Jupiters, typical values of A are a few $10^{-4}$. The amplitude of the signal is especially strong for planets having a high temperature (and thus being close to their star), a large radius and a low molecular weight.

For a temperate Jupiter with an equilibrium temperature of $400 \mathrm{~K}$ around a solar-type star, we find $A=7.7510^{-5}$. As compared with a hot Jupiter $(T=1600 \mathrm{~K})$, the amplitude is lower by a factor of 4 due to the low temperature. As compared with a super-Earth with a density equal of 4 times the Jovian value, with an effective temperature of $1600 \mathrm{~K}$, the amplitude of a temperate giant exoplanet is expected to be of the same order. Temperate Jupiters could thus be considered to exist in the detectability range of ARIEL.

\section{Secondary transit spectroscopy of a temperate Jupiter}

Secondary transits provide a direct measurement of the dayside emission of the exoplanet. The ratio of the planet/star flux can be approximated as follows (Tinetti et al. 2013):

- in the visible and near-infrared range:

$\rho 1=\left[\mathrm{R}_{\mathrm{P}} / \mathrm{R}^{*}\right]^{2} \times\left[\mathrm{T}_{\mathrm{P}} / \mathrm{T}^{*}\right]^{4}$

- in the mid and far-infrared (Rayleigh-Jeans approximation):

$\rho 2=\left[\mathrm{R}_{\mathrm{P}} / \mathrm{R}^{*}\right]^{2} \times\left[\mathrm{T}_{\mathrm{P}} / \mathrm{T}^{*}\right]$

For a temperate Jupiter $(\mathrm{T}=400 \mathrm{~K})$, as compared to a hot Jupiter $(\mathrm{T}=1600 \mathrm{~K}), \rho 1$ is lower by a factor $4^{4}=256$, and $\rho 2$ by a factor of 4 . As compared with a hot super-Earth, $\rho 1$ is lower by a factor of 10 and $\rho 2$ larger by a factor of 7.5. These numbers show that, in the near-infrared range, temperate Jupiters are not suitable targets for secondary transit spectroscopy. The comparison would be more favorable for $\rho 2$; however the Rayleigh-Jeans approximation holds only at long wavelengths (above about $20 \mu \mathrm{m}$ ), and is not valid in the spectral range of ARIEL $(2-8 \mu \mathrm{m})$.

It can be noted that the secondary transit spectrum of a temperate Jupiter could be significantly more complex than the primary transit spectrum, for two reasons: (1) if a temperature inversion takes place above the tropopause, the bands of $\mathrm{CH}_{4}, \mathrm{NH}_{3}$ and $\mathrm{H}_{2} \mathrm{O}$ could be present in emission; (2) photo-dissociation products of methane (i.e. $\mathrm{C}_{2} \mathrm{H}_{2}$, 
$\mathrm{C}_{2} \mathrm{H}_{6}$ ) could have strong signatures in the mid-infrared, as observed in the case of solar system giant planets.

In summary, we consider that, within the spectral range of ARIEL, temperate Jupiters are suitable targets for primary transit spectroscopy only.

\section{How to optimize the primary transit spectroscopy of temperate Jupiters}

We now consider temperate giant exoplanets orbiting low-mass stars. The advantage is twofold: (1) the amplitude of the primary transit signal is larger by a factor $\left[1 / R^{*}\right]^{2}$ where $\mathrm{R}^{*}$ is the radius of the star; (2) the revolution period is shorter than a year, which allows repeated transits of the object during the lifetime of the ARIEL mission.

For a star of mass $\mathrm{M}^{*}$ and radius $\mathrm{R}^{*}$ (in solar units) and effective temperature $\mathrm{T}^{*}$ (in $\mathrm{K}$ ), the star-planet distance D (in AU) of a fast-rotating exoplanet with an equilibrium temperature $\mathrm{T}_{\mathrm{P}}$ is given by the following equation:

$\left.\mathrm{D}=\left[(1-\mathrm{a})^{0.25} \times 279.0\left(\mathrm{~T}^{*} / 5770\right) \times \mathrm{R}^{* 0.5}\right] / \mathrm{T}_{\mathrm{P}}\right]^{2}$

or, using the equation

$\mathrm{L}^{*}=4 \Pi \sigma \mathrm{T}^{* 4} \mathrm{R}^{* 2}=\left(\mathrm{M}^{*} / \mathrm{Ms}\right)^{3}$

D can also be expressed as:

$\mathrm{D}=\left[(1-\mathrm{a})^{0.25} \times 279.0\left(\mathrm{M}^{*}\right]^{3 / 4} / \mathrm{T}_{\mathrm{P}}\right]^{2}$

where $\mathrm{a}$ is the albedo of the planet and $\mathrm{T}_{\mathrm{P}}$ its equilibrium temperature; $\mathrm{M}^{*}, \mathrm{R}^{*}$ and $\mathrm{T}^{*}$ are the mass, radius and effective temperature of the host star, expressed in solar units.

In the case of a slow-rotating (tidally locked) planet, equations (13) an (15) become:

$\left.\mathrm{D}=\left[(1-\mathrm{a})^{0.25} \times 331.0\left(\mathrm{~T}^{*} / 5770\right) \times \mathrm{R}^{* 0.5}\right] / \mathrm{T}_{\mathrm{P}}\right]^{2}$

and

$\mathrm{D}=\left[(1-\mathrm{a})^{0.25} \times 331.0\left(\mathrm{M}^{*}\right]^{3 / 4} / \mathrm{T}_{\mathrm{P}}\right]^{2}$

Assuming for our exoplanet two possible values of $\mathrm{T}_{\mathrm{P}}(350 \mathrm{~K}$ and $500 \mathrm{~K})$, we determine in each case its distance to its host star D. For each spectral type, we consider the two cases (fast rotating or tidally locked object) and we compare the two values of D with


while fast-rotating objects are expected in all other cases. Then we calculate the revolution period $\mathrm{P}$ of the exoplanet, using the equation:

$\mathrm{D}^{3} / \mathrm{P}^{2}=\mathrm{M}^{*}$

where $\mathrm{D}$ is in $\mathrm{AU}, \mathrm{P}$ in year and $\mathrm{M}^{*}$ in solar mass. 
We then calculate the amplitude of the transmission spectrum using Equation (9) for the two values of the exoplanet's equilibrium temperature. The transit time $t$ is estimated using the following equation:

$\mathrm{t}=\left[2 \mathrm{R}^{*} / \mathrm{D}\right] \times \mathrm{P} / 2 \Pi$

with $t$ expressed in hours, $\mathrm{P}$ in days, $\mathrm{R}^{*}$ in solar radii, and $\mathrm{D}$ in $\mathrm{AU}$, this equation becomes:

$t=0.0359 \times R^{*} \times P / D$

Table 2 lists the result for a variety of stars, ranging from solar-type stars to M8 dwarfs. 


\section{Table 2}

Estimated semi-major axis, rotational period, amplitude of primary transit signal and transit time for a Jovian-like exoplanet transiting around a star of spectral type between G2 and M8, using Equations (9), (13), (15), (16), (17), (18) and (20), with an albedo a = 0.03 , assuming either a fast rotator (columns 6 and 8) or a tidally locked object (columns 7 and 9). Two cases are considered: $\mathrm{T}_{\mathrm{P}}=350 \mathrm{~K}$ and $\mathrm{T}_{\mathrm{P}}=500 \mathrm{~K}$. The fast rotator case is favored for G2 to M0 stars (M0 stars are actually an intermediate case); the tidally locked object case is favored for M5 and M8 stars.

\begin{tabular}{|c|c|c|c|c|c|c|c|c|c|c|}
\hline Spectral type & $\begin{array}{l}\mathrm{R} \\
\text { (Rs) }\end{array}$ & $\begin{array}{l}\text { M } \\
(\mathrm{Ms})\end{array}$ & $\begin{array}{l}\mathrm{L} \\
\text { (Ls) }\end{array}$ & $\begin{array}{l}\mathrm{T}^{*} \\
(\mathrm{~K})\end{array}$ & $\begin{array}{l}\text { D } \\
(\mathrm{AU}) \\
\text { fast } \\
\text { rot. }\end{array}$ & $\begin{array}{l}\text { D } \\
(A U) \\
\text { slow } \\
\text { rot. }\end{array}$ & $\begin{array}{l}P(d) \\
\text { fast } \\
\text { rot. }\end{array}$ & $\begin{array}{l}P(d) \\
\text { slow } \\
\text { rot. }\end{array}$ & A & $\begin{array}{l}\text { Transit } \\
\text { time } \\
\text { (h) }\end{array}$ \\
\hline $\begin{array}{l}\mathrm{G} 2\left(\mathrm{~T}_{\mathrm{P}}=350 \mathrm{~K}\right) \\
\mathrm{G} 2\left(\mathrm{~T}_{\mathrm{P}}=500 \mathrm{~K}\right)\end{array}$ & 1.0 & 1.0 & 1.0 & 5770 & $\begin{array}{l}0.625 \\
0.306\end{array}$ & $\begin{array}{l}0.880 \\
0.431\end{array}$ & $\begin{array}{l}180 \\
61\end{array}$ & $\begin{array}{l}301 \\
103\end{array}$ & $\begin{array}{l}6.7810^{-5} \\
9.6910^{-5}\end{array}$ & $\begin{array}{l}10.3 \\
7.1\end{array}$ \\
\hline $\begin{array}{l}\mathrm{G5}\left(\mathrm{T}_{\mathrm{P}}=350 \mathrm{~K}\right) \\
\mathrm{G} 5\left(\mathrm{~T}_{\mathrm{P}}=500 \mathrm{~K}\right)\end{array}$ & 0.93 & 0.93 & 0.79 & 5641 & $\begin{array}{l}0.561 \\
0.274\end{array}$ & $\begin{array}{l}0.790 \\
0.386\end{array}$ & $\begin{array}{l}159 \\
54\end{array}$ & $\begin{array}{l}266 \\
91\end{array}$ & $\begin{array}{l}7.8410^{-5} \\
1.1210^{-4}\end{array}$ & $\begin{array}{l}9.4 \\
6.6\end{array}$ \\
\hline $\begin{array}{l}\mathrm{K} 0\left(\mathrm{~T}_{\mathrm{P}}=350 \mathrm{~K}\right) \\
\mathrm{K} 0\left(\mathrm{~T}_{\mathrm{P}}=500 \mathrm{~K}\right)\end{array}$ & 0.85 & 0.78 & 0.40 & 4977 & $\begin{array}{l}0.431 \\
0.211 \\
\end{array}$ & $\begin{array}{l}0.607 \\
0.297 \\
\end{array}$ & $\begin{array}{l}117 \\
40\end{array}$ & $\begin{array}{l}195 \\
67 \\
\end{array}$ & $\begin{array}{l}9.3810^{-5} \\
1.3410^{-4} \\
\end{array}$ & $\begin{array}{l}8.3 \\
5.8\end{array}$ \\
\hline $\begin{array}{l}\mathrm{K} 5\left(\mathrm{~T}_{\mathrm{P}}=350 \mathrm{~K}\right) \\
\mathrm{K} 5\left(\mathrm{~T}_{\mathrm{P}}=500 \mathrm{~K}\right)\end{array}$ & 0.74 & 0.69 & 0.16 & 4242 & $\begin{array}{l}0.358 \\
0.175\end{array}$ & $\begin{array}{l}0.504 \\
0.246\end{array}$ & $\begin{array}{l}94 \\
32\end{array}$ & $\begin{array}{l}157 \\
54\end{array}$ & $\begin{array}{l}1.2410^{-4} \\
1.7710^{-4}\end{array}$ & $\begin{array}{l}7.0 \\
4.9\end{array}$ \\
\hline $\begin{array}{l}M 0\left(T_{P}=350 \mathrm{~K}\right) \\
M 0\left(T_{P}=500 \mathrm{~K}\right)\end{array}$ & 0.63 & 0.47 & 0.063 & 3642 & $\begin{array}{l}0.201 \\
0.099 \\
\end{array}$ & $\begin{array}{l}0.282 \\
0.139 \\
\end{array}$ & $\begin{array}{l}48 \\
17\end{array}$ & $\begin{array}{l}80 \\
28\end{array}$ & $\begin{array}{l}1.7110^{-4} \\
2.4410^{-4}\end{array}$ & $\begin{array}{l}5.4 \\
3.9\end{array}$ \\
\hline $\begin{array}{l}\mathrm{M} 5\left(\mathrm{~T}_{\mathrm{P}}=350 \mathrm{~K}\right) \\
\mathrm{M} 5\left(\mathrm{~T}_{\mathrm{P}}=500 \mathrm{~K}\right)\end{array}$ & 0.32 & 0.21 & 0.008 & 3041 & $\begin{array}{l}0.06 \\
0.03 \\
\end{array}$ & $\begin{array}{l}0.08 \\
0.04\end{array}$ & $\begin{array}{l}12 \\
4 \\
\end{array}$ & $\begin{array}{l}18 \\
6 \\
\end{array}$ & $\begin{array}{l}6.6410^{-4} \\
9.5010^{-4} \\
\end{array}$ & $\begin{array}{l}2.6 \\
1.7 \\
\end{array}$ \\
\hline $\begin{array}{l}M 8\left(T_{P}=350 \mathrm{~K}\right) \\
M 8\left(T_{P}=500 \mathrm{~K}\right)\end{array}$ & 0.13 & 0.10 & 0.001 & 2691 & $\begin{array}{l}0.02 \\
0.01 \\
\end{array}$ & $\begin{array}{l}0.03 \\
0.015 \\
\end{array}$ & $\begin{array}{l}3 \\
1\end{array}$ & $\begin{array}{l}6 \\
2\end{array}$ & $\begin{array}{l}3.9810^{-3} \\
5.7010^{-3}\end{array}$ & $\begin{array}{l}1.0 \\
0.6\end{array}$ \\
\hline
\end{tabular}

\section{Sensitivity estimate}

In order to estimate the observing time needed to obtain a transmission spectrum of a temperate Jupiter, we use as a calibrator the exoplanet WASP-76 b, for which a synthetic transmission spectrum is shown in the ARIEL proposal. This object has a mass of 0.92 $\mathrm{M}_{\mathrm{J}}$, a radius of $1.83 \mathrm{R}_{\mathrm{J}}$, a temperature of $2200 \mathrm{~K}$. The star WASP-76, of V magnitude 9.5, located at $120 \mathrm{pc}$ from the Sun, has a mass of $1.46 \mathrm{Ms}$, a radius of $1.73 \mathrm{Rs}$, and a temperature of $6250 \mathrm{~K}$. The star-planet distance is $0.033 \mathrm{AU}$ and the rotation period of the exoplanet is 1.81 days. The amplitude of primary transit is about $1.010^{-3}$. The time transit is 3.4 hours. A summation of 25 transits (corresponding to 85 hours of total observing time) is needed to achieve a $\mathrm{S} / \mathrm{N}$ of about 10 .

We now estimate the number of transits needed for a temperate Jupiter around a Mdwarf to be observed by transmission spectroscopy with a total observing time of 100 hours. This corresponds to a S/N of about 12 for WASP-76 b, and about 2.5, 9.5 and 57 for temperate Jupiters around M0, M5 and M8 stars respectively. Table 3 summarizes the results. We also indicate the total time in orbit needed to accumulate the required 
number of transits; this time must be shorter than the lifetime of the ARIEL mission of 4 years. The case of WASP-76 is added for comparison.

\section{Table 3}

Number of transits required to obtain 100 hours of integration time on a temperate Jupiter around a M star and total time required to accumulate these transits.

\begin{tabular}{|l|l|l|l|l|l|l|l|}
\hline Spectral type & $\mathrm{R}^{*}(\mathrm{Rs})$ & $\mathrm{A}$ & $\begin{array}{l}\mathrm{S} / \mathrm{N} \text { in } \\
100 \text { hours }\end{array}$ & $\begin{array}{l}\text { Period } \\
(\mathrm{d})\end{array}$ & $\mathrm{t}(\mathrm{h})$ & $\begin{array}{l}\text { Number } \\
\text { of } \\
\text { transits }\end{array}$ & $\begin{array}{l}\text { Total time } \\
\text { needed for } \\
\mathrm{t}_{\text {tot }}=100 \mathrm{~h}(\mathrm{~d})\end{array}$ \\
\hline WASP-76 & 1.73 & $1.010^{-3}$ & 12 & 1.81 & 3.4 & 30 & 54 \\
\hline $\mathrm{M} 0\left(\mathrm{~T}_{\mathrm{P}}=350 \mathrm{~K}\right)$ & 0.63 & $1.7110^{-4}$ & 2 & 48 & 5.4 & 19 & 912 \\
$\mathrm{M} 0\left(\mathrm{~T}_{\mathrm{P}}=500 \mathrm{~K}\right)$ & & $2.4410^{-4}$ & 3 & 17 & 3.9 & 26 & 442 \\
\hline $\mathrm{M} 5\left(\mathrm{~T}_{\mathrm{P}}=350 \mathrm{~K}\right)$ & 0.32 & $6.6410^{-4}$ & 8 & 18 & 2.6 & 38 & 684 \\
$\mathrm{M} 5\left(\mathrm{~T}_{\mathrm{P}}=500 \mathrm{~K}\right)$ & & $9.5010^{-4}$ & 11 & 6 & 1.7 & 59 & 354 \\
\hline $\mathrm{M} 8\left(\mathrm{~T}_{\mathrm{P}}=350 \mathrm{~K}\right)$ & 0.13 & $3.9810^{-3}$ & 47 & 6 & 1.0 & 100 & 600 \\
$\mathrm{M} 8\left(\mathrm{~T}_{\mathrm{P}}=500 \mathrm{~K}\right)$ & & $5.7010^{-3}$ & 67 & 2 & 0.6 & 167 & 334 \\
\hline
\end{tabular}

It can be seen that all three classes of objects can be considered within a lifetime of 4 years; however, temperate Jupiters around M5 to M8 dwarfs should be favored for a higher S/N ratio and a shorter required time of observation. As shown in Fig. 4, a signalto-noise of 10 or higher with the resolving power of ARIEL $(100-200)$ should be sufficient for separating the signatures of the main molecular species $\left(\mathrm{H}_{2} \mathrm{O}, \mathrm{CH}_{4}, \mathrm{NH}_{3}, \mathrm{CO}\right.$, $\left.\mathrm{CO}_{2}\right)$.

\section{How to find exoplanets around M-dwarfs?}

So far we know of no example of temperate Jupiters around M stars. Can we estimate the probability of finding such target in the coming decade? We know that M-dwarfs are dominant in stellar populations, however they are difficult to identify because of their low luminosity. According to some authors (Henry et al. 2002; Reylé 2006), 70\% of these systems (corresponding to about 3000 samples) might exist remaining to be detected within 25 pc from the Sun. Several hundred of objects have already been found using the ground-based infrared surveys DENIS and 2MASS (Reylé 2006, Rajpurohit et al. 2014).

In addition, the CHEOPS, TESS and PLATO space missions are expected to provide new targets for transit spectroscopy. However, they will be oriented rather toward bright stars and small exoplanets. More importantly, the GAIA space mission is expected to bring an enormous database for this study. According to a simulation of stars observable with GAIA performed by Robin et al. (2014), a total of $1.310^{7}$ stars with a visual magnitude less than 12 (corresponding to the sensitivity limit of ARIEL) are expected to be observed by GAIA, including $11 \%$ of M-type stars. Knowing that about each star is expected to have a planet, the number of potential candidates is expected to be huge. In addition, GAIA will be able to perform photometry transits of Jupiter-like exoplanets around stars brighter than the $14^{\text {th }}$ magnitude, including M-type stars. 
It should be noted that transit spectroscopy around a M-dwarf star might be more difficult than around a $\mathrm{G}$ or $\mathrm{K}$ star, because of the intermittent flares emitted by these stars. However, the continuous near-infrared monitoring of the stellar flux by ARIEL, in parallel with the transit measurements, should make possible the identification and the removal of these anomalies.

Assuming M-stars can be found in sufficient numbers, one may question the probability of finding giant planets around such low-mass stars. We have no answer to this question yet, but some lessons can be drawn on the basis of the present exoplanet catalogs (Schneider, 2010). We know of about 150 exoplanets with masses greater than 10 jovian masses orbiting F, G or K stars, which seems to indicate that the mass ratio between a giant planet and its host star may actually be greater than the Jupiter/Sun mass ratio; a few examples of such objects can be found in Table 1. In addition, the recent detection of 7 planets in orbit around the ultra-cool M8 star TRAPPIST-1 (Gillon et al. 2017), possibly the result of a migration from beyond the ice line, may suggest that the presence of larger objects is also possible. In addition, the exoplanet demographics from microlensing suggest that giant planets around M-stars are more frequent than expected from radio-velocimetry studies, with a frequency of 0.11 for planets more massive than 50 terrestrial masses (Clanton and Gaudi, 2014).

\section{Conclusion}

In summary, it appears that temperate Jupiters orbiting $M$ stars at semi-major axes between about 0.1 and $0.5 \mathrm{AU}$ could be suitable targets for ARIEL. If such targets are identified in future surveys, their study with ARIEL would allow us to enlarge the range of physical atmospheric parameters to be covered, and thus to extend the exoplanets' exploration by the mission.

\section{References}

Bolton, S. J. et al. 2017. Jupiter's interior and deep atmosphere: The initial pole-to-pole passes with the Juno spacecraft. Science 356, 6340-6344

Clanton, C. and Gaudi, B. S. 2014. Synthetizing exoplanet demographics from radial velocity and microlensing surveys. II. The frequency of planets orbiting M-dwarfs. Astrophys. J. 791:91 (23pp)

Encrenaz, T. et al. 1999. The atmospheric composition and structure of Jupiter and Saturn from ISO observations: What have we learnt? Plan. Space Sci. 47, 1225-1242

Gillon, M. et al. 2017. Seven temperate terrestrial planets around the nearby utra-cool dwarf star TRAPPIST-1. Nature 542, 456-460

Grevesse, N., Asplund, M., Sauval, A.J., 2005. The new solar chemical composition. In: Alecian, G., Richard, O., Vauclair, S. (Eds.), Element Stratification of Stars, 40 years of Atomic Diffusion, EAS Publication Series, EDP Sciences. 
Henry, T, Walkowicz, L. M., Barto, T. C., Golimowsky, D. A. 2002. Astron. J. 123, 2002

Kasting, J. F., Whitmire, D. P., and Reynolds, R. T. 1993. Habitable zones around mainsequence stars. Icarus 101, 108-128

Lainey, V. 2016. Quantification of tidal parameters from Solar system data.

Léger, A. et al. 2009. Transiting exoplanets from the CoRoT space mission. VIII CoRoT-7 b: the first super-Earth with measured radius. Astron. Astrophys. 506, 287-302

Morley, C. V. et al. 2012. Neglected clouds in T and Y dwarf atmospheres. Astrophys. J. 756:172(17pp)

Murray, C. D., Dermott, S. F. 2000. Solar system dynamics, Cambride University Press

Owen, T. and Encrenaz, T. 2006. Compositional constraints on giant planet formation. Plan. Space Sci. 54, 1188-1196

Pollack, J. B. et al. 1996. Formation of the giant planets by concurrent accretion of solids and gas. Icarus 124, 62-85

Rajpurohit, A. S., Reylé, C., Allard, F. et al. 2013. Astron. Astrophys. 556, A15

Reylé C. 2006.Habilitation à Diriger des Recherches, Observatoire de Besançon.

Robin A et al. 2004

Robin, A., Reylé, C., Luri, X. et al. 2014. Mem. S. A. It. 85, 560

Sudarsky, D., Burrows, A., Pinto, P. 2000. Albedos and reflection spectra of extrasolar giant planets. Astrophys. J. 588, 1121-1148

Tinetti, G. et al. 2011.The EChO Mission Proposal - A candidate for the ESA M3 mission

Tinetti, G., Encrenaz, T., Coustenis, A. Spectroscopy of planetary atmospheres in our Galaxy. 2013. Astron. Astrophys. Rev. 21, 63

Tinetti, G. et al. 2015. The ARIEL Mission Proposal - A candidate for the ESA M4 mission 